Silva, DB, Silva, AAP, Muchalak, F, Brito, LHP \& Carfane, DG. (2020). Qualitative floristic survey and dispersion syndrome of native species of the cerrado. Research, Society and Development, 9(7):1-10, e288974236.

\title{
Levantamento florístico qualitativo e síndrome de dispersão de espécies nativas do
} cerrado

Qualitative floristic survey and dispersion syndrome of native species of the cerrado Levantamiento florística cualitativa y síndrome de dispersión de especies nativas del cerrado

Recebido: 05/05/2020 | Revisado: 06/05/2020 | Aceito: 06/05/2020 | Publicado: 12/05/2020

Dayane Bortoloto da Silva

ORCID: http://orcid.org/0000-0002-3264-0390

Universidade Estadual Paulista "Júlio de Mesquita Filho", Brasil

E-mail: dayebortoloto@gmail.com

Arlindo Ananias Pereira da Silva

ORCID: http://orcid.org/0000-0002-3093-6796

Universidade Estadual Paulista "Júlio de Mesquita Filho", Brasil

E-mail: arlindo.ananias@unesp.br

Franciele Muchalak

ORCID: http://orcid.org/0000-0001-8281-6350

Universidade Federal de Mato Grosso do Sul

E-mail: francieleddd@ hotmail.com

Leonardo Henrique Pereira Brito

ORCID: http://orcid.org/0000-0001-6875-0455

Universidade Estadual Paulista "Júlio de Mesquita Filho", Brasil

E-mail: leonardo.henrique17@hotmail.com

Douglas Garrio Carfane

ORCID: http://orcid.org/0000-0001-7226-2104

Universidade Estadual Paulista “Júlio de Mesquita Filho”, Brasil

E-mail: douglascarfane@gmail.com

\section{Resumo}

A dispersão de frutos e sementes é um dos diversos processos biológicos e físicos que ocorrem em florestas, proporcionando a manutenção das populações de espécies vegetais. 
Este processo é importante para a reprodução das plantas, sendo que o padrão de dispersão de sementes ajuda na determinação da área potencial de recrutamento das plantas e também serve como base para os processos subsequentes, como competição, predação e reprodução. $\mathrm{O}$ objetivo deste trabalho foi analisar a composição florística, investigar a ocorrência e a distribuição das síndromes de dispersão de frutos ou sementes. O presente estudo foi desenvolvido em Chapadão do Sul, MS, em área de cerrado antropizado. O levantamento florístico foi realizado através do método de parcelas quadradas, sendo amostrados todos os indivíduos arbóreos com DAP (diâmetro a altura do peito $=1,30 \mathrm{~m}$ do solo) $\geq 5 \mathrm{~cm}$, que se encontravam dentro das parcelas. Foram marcadas dez parcelas, medindo $20 \mathrm{~m}$ de largura por $20 \mathrm{~m}$ de comprimento, totalizando $4.000 \mathrm{~m}^{2}$ amostrados. Através desta classificação foram relacionadas às espécies em cada parcela. De todos os indivíduos amostrados foram coletadas amostras botânicas, montando exsicatas, e enviadas para identificação das mesmas. Foram encontrados indivíduos pertencentes a 32 espécies e 18 famílias botânicas. Das espécies amostradas 40,63\% apresentavam a anemocoria como estratégia de dispersão de seus frutos e sementes, 21,88\% autocoria e 37,5\% zoocoria. Apesar da dispersão anemocórica apresentar maior número de espécies, este valor é próximo da dispersão zoocórica, o que pode representar um estabelecimento de relações ecológicas entre flora e fauna local.

Palavras-chave: Área antropizada; Biologia reprodutiva; Relações ecológicas.

\section{Abstract}

The dispersion of fruits and seeds is one of several biological and physical processes that occur in forests, providing the maintenance of populations of plant species. This process is important for plant reproduction, and the seed dispersal pattern helps in determining the potential recruitment area of the plants and also serves as a basis for subsequent processes, such as competition, predation, and reproduction. This research aims to analise floristic composition, investigate occurrence and distribution of fruit or seed dispersal syndromes. The present study was carried out in Chapadão do Sul, MS, in an anthropized savannah area. The floristic survey was carried out using the square plot method, sampling all arboreal individuals with DBH (diameter at breast height $=1.30 \mathrm{~m}$ from the ground) $\geq 5 \mathrm{~cm}$, which was inside the plots. Ten plots were marked, measuring $20 \mathrm{~m}$ wide by $20 \mathrm{~m}$ long, totaling 4,000 $\mathrm{m} 2$ sampled. Through this classification, they were related to the species in each plot. Botanical samples were collected from all the individuals sampled, assembling desiccate, and sent to identify them. Individuals belonging to 32 species and 18 botanical families were found. Of the sampled species, $40.63 \%$ presented anemochory as a strategy for dispersing 
their fruits and seeds, $21.88 \%$ autochory, and 37.5\% zoochory. Although the anemochoric dispersion has a greater number of species, this value is close to the zoochoric dispersion, which may represent an establishment of ecological relationships between local flora and fauna.

Keywords: Anthropized area; Reproductive biology; Ecological relations.

\section{Resumen}

La dispersión de frutas y semillas es uno de varios procesos biológicos y físicos que ocurren en los bosques, proporcionando el mantenimiento de poblaciones de especies de plantas. Este proceso es importante para la reproducción de las plantas, y el patrón de dispersión de semillas ayuda a determinar el área potencial de reclutamiento de las plantas y también sirve como base para procesos posteriores, como la competencia, la depredación y la reproducción. El objetivo de este trabajo fue analizar la composición florística, investigar la ocurrencia y distribución de síndromes de dispersión de frutos o semillas. El presente estudio se llevó a cabo en Chapadão do Sul, MS, en un área de sabana antropizada. La encuesta florística se realizó utilizando el método de parcela cuadrada, muestreando a todos los individuos arbóreos con DAP (diámetro a la altura del pecho $=1.30 \mathrm{~m}$ del suelo) $\geq 5 \mathrm{~cm}$, que estaba dentro de las parcelas. Se marcaron diez parcelas, que miden $20 \mathrm{~m}$ de ancho por $20 \mathrm{~m}$ de largo, con un total de $4.000 \mathrm{~m} 2$ muestreados. A través de esta clasificación, se relacionaron con las especies en cada parcela. Se recolectaron muestras botánicas de todos los individuos muestreados, reuniéndose para desecar, y enviados para identificarlos. Se encontraron individuos pertenecientes a 32 especies y 18 familias botánicas. De las especies muestreadas, 40.63\% presentaron anemochory como una estrategia para dispersar sus frutos y semillas, $21.88 \%$ de autochory y $37.5 \%$ zoochory. Aunque la dispersión anemocórica tiene un mayor número de especies, este valor está cerca de la dispersión zoocorica, que puede representar un establecimiento de relaciones ecológicas entre la flora y la fauna local.

Palabras clave: Área antropizada; Biología reproductiva; Relaciones ecológicas.

\section{Introdução}

A evolução dos frutos é caracterizada por processos de seleção natural, que gerou espécies adaptadas a diversos ecossistemas terrestres, decorrente de milhares de anos de interação entre a flora e o ambiente (Haven et al., 2001). Esse é um aspecto de extrema 
importância na evolução das angiospermas, que ocorreu devido à adaptação ao seu agente dispersor (Saravy et al., 2003).

A dispersão de frutos e sementes é um dos diversos processos biológicos e físicos que ocorrem em florestas proporcionando a manutenção da população de espécies vegetais (Terborgh, 1990). O processo de dispersão é importante para a reprodução das plantas, pois a semente deve chegar a um local propício para germinar, com certa distância da planta-mãe, evitando a competição e também predadores de sementes e plantas que ficam na proximidade (Howe, 1993 e Janzen, 1970).

As dispersões de sementes podem ser divididas em três tipos, Anemocoria, sementes dispersas pelo vento, os frutos e, ou sementes, apresentam alas permitindo que seja levado de um local ao outro (Haven et al., 2001) como ocorre na espécie Machaerium acutifolium, em que seus frutos são indeiscentes e apresentam alas. Autocoria é a dispersão que ocorre pela própria planta (Pijl, 1982). Os frutos maduros arrebentam-se e as sementes são lançadas para longe das matrizes (Ducke, 1949), podendo também haver disseminação do fruto pelo seu próprio peso (Pijl, 1982), e depois dispersa por animais (Saravy et al., 2003). Zoocoria na qual a dispersão é feita por animais, após a semente ser passada pelo tubo digestivo do animal, sem ocorrer nenhum dano, são espalhadas (Haven et al., 2001). Neste caso pode há uma subdivisão, conforme a dieta de alguns animais, diszoocoria (roedores), ornitocoria (aves) e quiropterocoria (morcegos), entre outras (Saravy et al., 2003)

O padrão de dispersão de sementes ajuda na determinação da área potencial de recrutamento das plantas e também serve como base para os processos subsequentes, como competição, predação e reprodução (Nathan e Muller-Landau, 2000)

A dispersão de sementes é de extrema importância na distribuição e aumento da sobrevivência das plantas. Isto ocorre para que as sementes tenham maior chance de germinar e diminua a chance de competição com a árvores matriz (Saravy et al., 2003). Nesse sentido o objetivo deste trabalho foi analisar a composição florística, investigar a ocorrência e a distribuição das síndromes de dispersão de frutos ou sementes.

\section{Metodologia}

Uma pesquisa visa trazer novos conhecimentos ou saberes para a sociedade como preconiza Pereira et al. (2018) e os estudos e pesquisas em campo possuem mais variáveis que são difíceis de ser controladas em relação às pesquisas laboratoriais. O presente estudo foi desenvolvido em Chapadão do Sul, MS, em área de cerrado antropizado. O Município possui 
uma área de $3.851 \mathrm{~km} 2$, está localizado na porção nordeste do Estado de Mato Grosso do Sul e faz parte da Micro-Região Geográfica de Cassilândia. Sua sede está a uma altitude de 790 m acima do nível do mar e situa-se nas seguintes coordenadas geográficas: Latitude - $18^{\circ} 41^{\text {' }}$ 33" Sul e Longitude - 52 40' 45" Oeste de Greenwich, distanciando da capital 330 km.

A cobertura vegetal original do Município é de cerrados e campos limpos e a classe de solo predominante é o Latossolo Vermelho distrófico. O clima é, segundo Köppen, do tipo tropical úmido (Aw), com estação chuvosa no verão e seca no inverno e precipitação média anual de $1.850 \mathrm{~mm}$. A temperatura média anual varia de $13^{\circ} \mathrm{C}$ a $28^{\circ} \mathrm{C}$.

O levantamento florístico foi realizado por meio do método de parcelas quadradas, sendo amostrados todos os indivíduos arbóreos com DAP (diâmetro a altura do peito $=1,30 \mathrm{~m}$ do solo) $\geq 5 \mathrm{~cm}$, que se encontravam dentro das parcelas. Foram marcadas dez parcelas, medindo $20 \mathrm{~m}$ de largura por $20 \mathrm{~m}$ de comprimento, totalizando $4.000 \mathrm{~m}^{2}$ amostrados. Através desta classificação foram relacionadas às espécies em cada parcela. De todos os indivíduos amostrados foram coletadas amostras botânicas, montando exsicatas, e enviadas para identificação das mesmas.

\section{Resultados e Discussão}

Foram encontrados indivíduos pertencentes a 32 espécies e 18 famílias botânicas (Tabela 1).

Tabela 1. Espécies identificadas nas parcelas e síndrome de dispersão.

\begin{tabular}{|c|c|c|c|c|}
\hline FAMÍLIA & ESPÉCIE & $\begin{array}{c}\text { NOME } \\
\text { VULGAR }\end{array}$ & DISPERSÃO & AUTOR \\
\hline Anacardiaceae & Anacardium bumile Mart. & $\begin{array}{l}\text { Caju-do- } \\
\text { cerrado }\end{array}$ & Zoo & Saravy et al. (2003) \\
\hline Anonaceae & Annona crassiflora Mart. & Araticum & Auto & Ferreira (1980) \\
\hline \multirow[t]{2}{*}{ Apocynaceae } & $\begin{array}{c}\text { Aspidosperma macrocarpon } \\
\text { Mart. }\end{array}$ & Guatambu & Anemo & Saravy et al. (2003) \\
\hline & Hancornia speciosa Gomez & Mangaba & Zoo & \\
\hline Araliaceae & Schefflera macrocarpa C. \& S. & Maudioção & Zoo & Ohashi e Leão (2005) \\
\hline Asteraceae & $\begin{array}{c}\text { Piptocarpha rotundifolia } \\
\text { (Less.) Baker }\end{array}$ & $\begin{array}{l}\text { Coração-de- } \\
\text { negro }\end{array}$ & Anemo & Ferreira (1974) \\
\hline \multirow[t]{2}{*}{ Bignoniaceae } & $\begin{array}{c}\text { Cybistax antisyphilitca (Mart.) } \\
\text { Mart. }\end{array}$ & Ipê-verde & Anemo & Lorenzi (1992) \\
\hline & Tabebuia aurea (Mart.) Bur. & Ipê-amarelo & Anemo & Lorenzi (1992) \\
\hline \multirow{3}{*}{$\begin{array}{c}\text { Caryocaceae } \\
\text { Clusiaceae }\end{array}$} & Caryocar brasiliense Camb. & Pequi & Zoo & Barradas (1972) \\
\hline & Kielmeyera coriacea (Spr.) & Pau-santo & Anemo & Botello e Carneiro \\
\hline & & 5 & & \\
\hline
\end{tabular}


Research, Society and Development, v. 9, n. 7, e288974236, 2020

(CC BY 4.0) | ISSN 2525-3409 | DOI: http://dx.doi.org/10.33448/rsd-v9i7.4236

Mart.

(1992)

Fabaceae

Anadenanthera falcata (Benth.)

Speg.

Angico

Auto

Costa (1988)

Andira bumilis Mart. Ex Benth. Mata-barata

Auto

Rizzini (1971)

Bowdichia virgilioides Kunth. Sucupira-preta

Brandão e Ferreira

Copaifera langsdorffii Desf.

Copaíba

Auto

(1991)

Dimorphandra mollis Benth.

Faveira

Auto

Eira et al. (1992)

Barros e Caldas

(1980)

Hymenaea stigonocarpa Mart.

Ex Hayne

Jatobá

Zoo

Carneiro et al. (1976)

Machaerium acutifolium Vog.

Jacaranda

Anemo

Saravy et al. (2003)

Plathymenia reticulada Benth.

Vinhático

Anemo

Carneiro et al. (1976)

Stryphnodendron adstingens

(Vell.) Covile

Barbatimão

Auto

Lorenzi (2006)

Stryphnodendron barbadetiman

(Vell.) Covile

Borãozinho-

roxo

Auto

Barros e Caldas

Malpighiaceae

Malvaceae

Byrsonima verbascifolia Rich.

Murici

Zoo

Paineira

Anemo

Saravy et al. (2003)

Zucc.

Pseudobombax longiflorum

(Mart. \& Zucc.)

Imbiruçu

Anemo

Lorenzi (1992)

Nascimento e Saddi (1992)

Tibouchina papyrus (Pohl) Toledo

Quaresmeira Anemo

Almeida et al. (1998)

Machado et al. (1986)

Eugenia dysenterica Mart. Ex. DC.

Cagaita

Zoo

Saravy et al. (2003)

Proteaceae

Roupala montana Aubl. Alibertia edulis (A. Rich.) L. Rich.

Carne-de-vaca

Anemo

Almeida et al. (1998)

Rubiaceae

Marmelada

Zoo

Guarin neto (1986)

Sapotaceae

Pouteria ramiflora Radlk.

Curriola

Zoo Pouteria torta Radlk.

Grão-de-galo

Zoo

Almeida et. (1998)

Lorenzi (2006)

Vochysiaceae

Qualea grandiflora Mart.

Pau-terra

Anemo

Barbosa et al. (1985)

Qualea parviflora Mart.

Pau-terrinha

Anemo

Almeida et al. (1988)

Anemo: Anemocoria; Auto: Autocoria; Zoo: Zoocoria.

Fonte: próprios autores

Sendo que a família Fabaceae apresentou o maior número de espécies amostradas, dez espécies, seguido das demais famílias com duas ou uma espécie dentro de cada família.

Das espécies amostradas 40,63\% apresentavam a anemocoria como estratégia de dispersão de seus frutos e sementes, $21,88 \%$ autocoria e 37,5\% zoocoria (Grafico 1A). Diferentemente dos resultados encontrados por Zama et al., (2012) que realizando um levantamento florístico e estudando a síndrome de dispersão de espécies nativas do Parque 
Estadual Mata São Francisco, em Cornélio Procópio, onde a dispersão zoocórica, foi a que apresentou maior valor 64\%, seguidas pelas anemocórica, 23\%, e autocórica,13\%. Possivelmente por se tratar de um Parque Estadual onde não há um grande distúrbio, as relações ecológicas entre a fauna e flora estão estabelecidas, o que propicia um maior valor para dispersão zoocórica. Contrário da área deste estudo que apresenta uma grande intervenção antrópica, ocorrendo principalmente espécies pioneiras que possuem a anemocoria como estratégia de dispersão.

Dentro da dispersão anemocórica, a família Fabaceae, Bignoniaceae, Malvaceae, e Vochysiaceae apresentaram ambas 15,67\% (Gráfico 1B). Entretanto as demais famílias em que foi verificada esse tipo de dispersão representaram 38,36\%, não foi verificada espécies das famílias Anacardiaceae, Annonaceae, Araliaceae, Caryocaceae, Malpighiaceae, Moraceae, Myrtaceae, Rubiaceae e Sapotaceae apresentando este tipo de dispersão.

Das espécies que apresentaram autocoria como estratégia de dispersão de seus frutos e sementes, 85,71\% pertenciam a família Fabaceae e 14,29\% Anonaceae (Gárfico 1C), não foram verificados para as demais famílias. Dentro da dispersão zoocorica a família Fabaceae e Sapotaceae apresentaram ambas 16,67\%, e as demais 66,67\% (Gráfico 1D). Contudo não foi verificado este tipo de dispersão para as famílias Annonaceae, Apocynaceae, Asteraceae, Bignoniaceae, Clusiaceae, Malvaceae, Melastomataceae, Proteaceae e Vochysiaceae.

Gráfico 1. A) Distribuição das espécies dentro do tipo de dispersão. 1B) distribuição das famílias dentro da dispersão anemocórica. 1C) Distribuição das famílias dentro da dispersão autocórica e 1D) Distribuição das famílias dentro da dispersão zoocórica.
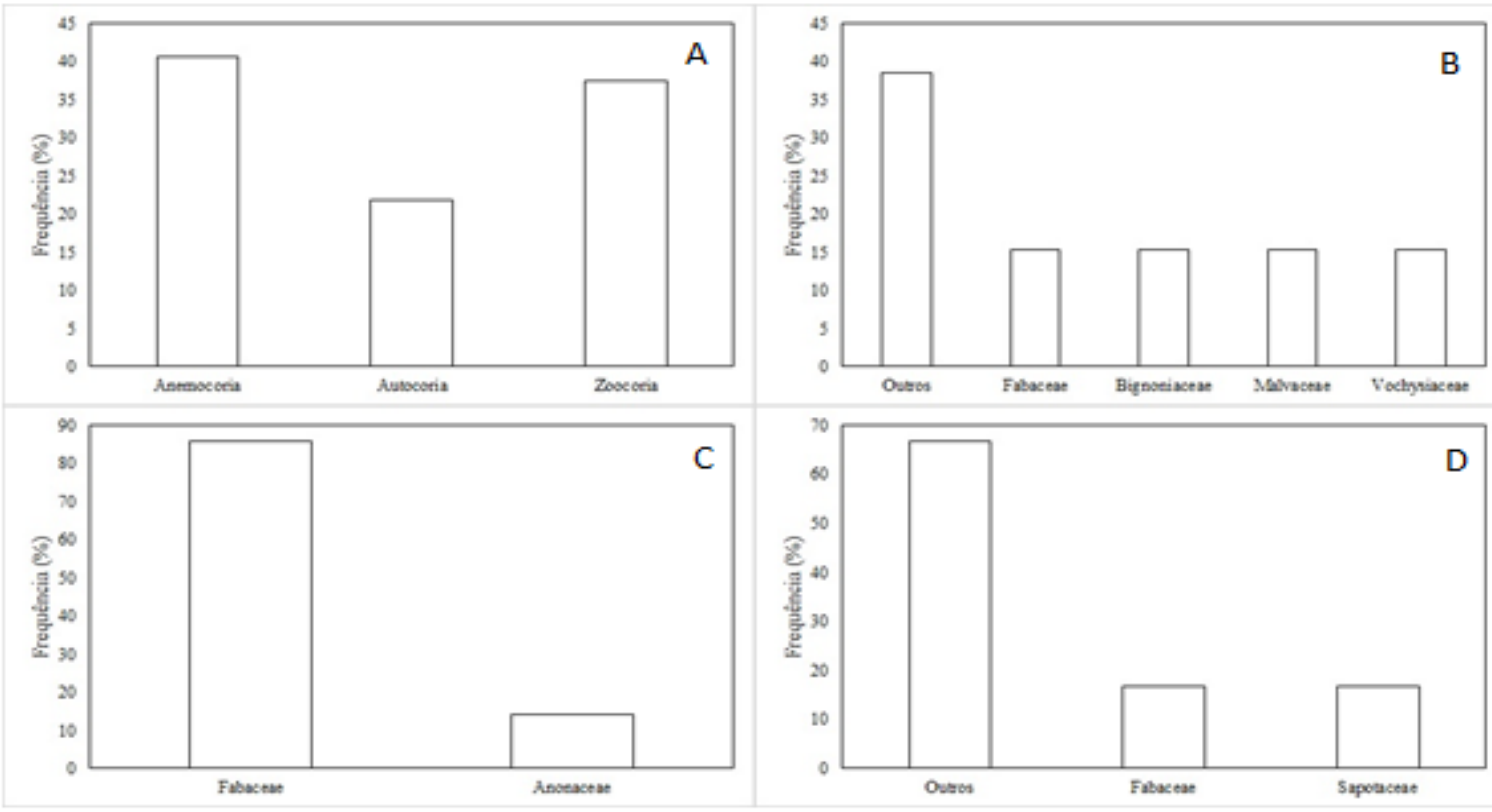


\section{Conclusão}

Apesar da dispersão anemocórica apresentar maior número de espécies, este valor é próximo da dispersão zoocórica, o que pode representar um estabelecimento de relações ecológicas entre flora e fauna local.

\section{Referências}

Ducke, A. (1949). Árvores Amazônicas e sua propagação: adaptação de frutos ou sementes de árvores amazônicas a diversos meios de propagação em espécies de estreita afinidade botânica, porém em condições mesológicas diferentes. Boletim do Museu Paraense Emílio Goeldi. (10), 81-92.

Haven, PH, Evert, RF, Eichhorn, SE. (2001). Biologia Vegetal. 6.ed. Rio de Janeiro: Guanabara Koogan. 522-527.

Howe, HF. (1993). Aspects of variation in a neotropical seed dispersal system. In Frugivory and seed dispersal: ecological and evolutionary aspects. Springer, Dordrecht. 149-162.

Janzen, DH. (1970). Herbivores and the number of tree species in tropical forests. The American Naturalist, 104(940), 501-528.

Nathan, R \& Muller-Landau, HC. (2000). Spatial patterns of seed dispersal, their determinants and consequences for recruitment. Trends in ecology \& evolution, 15(7), 278285.

Pereira, AS et al. (2018). Metodologia da pesquisa científica. [e-book]. Santa Maria. Ed. UAB/NTE/UFSM. Acesso em: 10 maio 2020. Disponível em: https://repositorio.ufsm.br/bitstream/handle/1/15824/Lic_Computacao_MetodologiaPesquisa-Cientifica.pdf?sequence $=1$. 
Pijl, L. (1982). Principles of dispersal in higher plants. Berlin: Springer Verlag. 215 p.

Saravy, FP, Freitas, PD, Lage, MA, Leite, SJ, Braga, LF \& Sousa, MP. (2003). Síndrome de dispersão em estratos arbóreos em um fragmento de floresta ombrófila aberta e densa em alta floresta-MT. Revista do Programa de Ciências Agro-Ambientais, 2(1), 1-12.

Terborgh, J. (1990). Seed and fruit dispersal-Commentary. In: Reproductive ecology of tropical forest plants. Paris: The Parthenon Publishing Group. (7) 181-190.

Zama, MY, Bovolenta, YR, Carvalho, EDS, Rodrigues, DR, Araujo, CGD, Sorace, MADF \& Luz, DG. (2012). Florística e síndromes de dispersão de espécies arbustivo-arbóreas no Parque Estadual Mata São Francisco, PR, Brasil. Hoehnea, 39(3), 369-378.

\section{Porcentagem de contribuição de cada autor no manuscrito}

Dayane Bortoloto da Silva $-25 \%$

Arlindo Ananias Pereira da Silva - 25\%

Franciele Muchalak - 18\%

Leonardo Henrique Pereira Brito - $16 \%$

Douglas Garrio Carfane - $16 \%$ 